\title{
Study of electroweak processes in the two and three-nucleon systems with local chiral forces
}

\author{
Roman Skibinski*; Jacek Golak, Kacper Topolnicki, Henryk Witała, Alaa E. \\ Elmeshneb \\ M.Smoluchowski Institute of Physics, Jagiellonian University, 30-348, Kraków, Poland \\ E-mail: roman.skibinski@uj.edu.pl
}

\begin{abstract}
Deuteron photodisintegration, three-body photodisintegration of ${ }^{3} \mathrm{He}$ as well as muon capture on the deuteron and ${ }^{3} \mathrm{He}$ are theoretically analyzed by means of improved chiral nucleon-nucleon interactions. To this end we solve the Schrödinger and Faddeev equations in the momentum space. While our electromagnetic current operator implicitly receives implicitly many-body contributions via the Siegert theorem, the weak current is taken in the form of the nonrelativistic single nucleon current supplemented by the dominant relativistic corrections. Such models of the current operators and the wave functions generated with the new chiral potentials lead to reasonable predictions for the cross sections and capture rates studied in this contribution. Our results show very welcome features of the new forces and prove their usefulness in studies of electroweak processes with few nucleons.
\end{abstract}

The 8th International Workshop on Chiral Dynamics

29 June 2015 - 03 July 2015

Pisa, Italy

\footnotetext{
* Speaker.

$\dagger$ The authors would like to thank Dr L.E.Marcucci for her help and discussions on the weak current operator. This work was supported by the Polish National Science Center under Grant No. DEC-2013/10/M/ST2/00420. Some part of the work was performed under the LENPIC collaboration. The numerical calculations have been performed on the supercomputer cluster of the JSC, Jülich, Germany.
} 


\section{Introduction}

Chiral Effective Field Theory ( $\chi$ EFT) is a powerful theoretical tool which allows one to derive consistent nuclear forces and electroweak current operators. Its connection to $\mathrm{QCD}$, via symmetries of the underlying Lagrangian, makes it the well founded theory of nuclear interactions. The strong development of $\chi \mathrm{EFT}$ in recent years has resulted in more and more sophisticated models of the nucleon-nucleon $(\mathrm{NN})$ potential $[1,2] . \chi \mathrm{EFT}$ can be applied in many variant forms, like the pionless version [3] or the theory with explicit treatment of the Delta resonance [4]. However, the most successful approach to the NN interaction is based on only nucleonic and pionic degrees of freedom. Nowadays it allows one to describe $\mathrm{NN}$ scattering at energies up to $300 \mathrm{MeV}$. It can be also extended to many-nucleon interactions and indeed investigations of the chiral three-nucleon force $(3 \mathrm{NF})$ as well as the four-nucleon potential are ongoing. One of the inherent features of the nuclear force models derived from $\chi \mathrm{EFT}$ is the necessity of regularization of the obtained forces. The regularization in the momentum space with the non-local regulator was used for a long time [5]. It was shown recently [6], that such a regularization scheme introduces too many artifacts and destroys the correct physical behavior of the potential at long distances. In addition, the spectral function regularization introduced an unwanted short-range part of the two-pion exchange potential. That causes too strong dependence of predictions on values of the cut-off parameters [6].

The Bochum-Bonn group proposed recently in $[7,8]$ an improved version of the chiral potential up to fifth order of the chiral expansion $\left(\mathrm{N}^{4} \mathrm{LO}\right)$. During its construction regularization in the coordinate space is performed and only then is, such a regularized force, transformed to the momentum space. Using this procedure different partial waves are affected by the regularization in a different manner, what in turn leads to smaller undesirable regularization effects. Indeed, as shown in $[7,8]$ the NN phase shifts as well as the deuteron properties are much less sensitive to the values of the regularization parameter than the older model [5]. The same is also true for the elastic nucleon-deuteron $(\mathrm{Nd})$ scattering observables. The neutron-deuteron (nd) differential cross section at $\mathrm{E}=10,70,150$ and $200 \mathrm{MeV}$ presented in [9] shows only weak dependence on the regularization parameter R. It is totally negligible at lower energies and becomes visible only at higher energies.

In this paper we investigate if the same observation is valid for electromagnetic and weak processes. Note that for these processes the electromagnetic and weak currents have to be taken into account. Unfortunately, they are yet not developed at the same orders and beside the well known single nucleon current (SNC), only electromagnetic two-nucleon ( $2 \mathrm{~N}$ ) currents at LO and some of the leading NLO corrections are available [10]. Together with the old, nonlocal version of chiral interaction they were used to study the deuteron and the ${ }^{3} \mathrm{He}$ photodisintegration in Ref. [11, 12]. While the general description of observables was reasonable, a strong cut-off dependence of predictions was also observed. Similar results have been obtained for the radiative nucleon-deuteron capture and ${ }^{3} \mathrm{He}$ photodisintegration reactions when instead of explicit many-body currents the Siegert theorem was used [13]. The too big spread of predictions with different values of cut-off parameters practically disallowed us drawing physical conclusions. Thus it is very interesting to see if the cut-off dependence becomes smaller when the improved model of chiral interactions is used.

We also apply the new NN forces to studies of weak muon capture reactions on the deuteron and ${ }^{3} \mathrm{He}$, focusing on the cut-off dependence of the capture rates. In this paper we restrict ourselves 
to calculations in which the weak current is taken as a nonrelativistic single nucleon current operator supplemented with leading relativistic corrections (RC) [14]. Since we are mainly interested in the cut-off dependence of different observables, the use of the incomplete model of the current operator is justified in this work.

In the next chapter we give a short overview of our formalism for electromagnetic and weak processes. Chapter 3 contains selected results for the cross sections in $\gamma+\mathrm{d} \rightarrow \mathrm{p}+\mathrm{n}, \gamma+{ }^{3} \mathrm{He} \rightarrow$ $\mathrm{p}+\mathrm{p}+\mathrm{n}, \mu^{-}+{ }^{2} \mathrm{H} \rightarrow \mathrm{n}+\mathrm{n}+v_{\mu}$ and $\mu^{-}+{ }^{3} \mathrm{He} \rightarrow{ }^{3} \mathrm{H}+v_{\mu}$ reactions. We conclude in Chapter 4.

\section{Formalism}

The present study is based on the formalism described in detail in $[15,16,17,18,11]$, so here we display only the main steps. We work in the momentum space and use formalism of the Faddeev equations. The nuclear matrix elements for electromagnetic or weak disintegrations are central quantities from which we are able to calculate observables [17].

In the case of deuteron photodisintegration the nuclear matrix element $N_{\mathrm{deu}}^{\mu}$ is defined as

$$
N_{\text {deu }}^{\mu} \equiv\left\langle\Psi_{\text {scatt }}^{2 \mathrm{~N}}\left|j^{\mu}\right| \Psi_{\text {bound }}^{2 \mathrm{~N}}\right\rangle,
$$

where $\left|\Psi_{\text {scatt }}^{2 \mathrm{~N}}\right\rangle$ and $\left|\Psi_{\text {bound }}^{2 \mathrm{~N}}\right\rangle$ are the final proton-neutron scattering state and the initial deuteron bound state, respectively. The deuteron state is obtained by solving the Schrödinger equation with the Hamiltonian comprising the $2 \mathrm{~N}$ interaction $V$. The same interaction, together with the free $2 \mathrm{~N}$ propagator $G_{0}$, enters the Lippmann-Schwinger equation for the $t$ operator, $t=V+t G_{0} V$ which we use to obtain the final scattering state. Namely, $N_{\text {deu }}^{\mu}$ can be expressed as

$$
N_{\text {deu }}^{\mu}=\left\langle\vec{p}_{0}\left|\left(1+t G_{0}\right) j^{\mu}\right| \Psi_{\text {bound }}^{2 \mathrm{~N}}\right\rangle,
$$

where $\left|\vec{p}_{0}\right\rangle$ is the eigenstate of the relative proton-neutron momentum. The details of used electromagnetic current operator $j^{\mu}$ are discussed below.

Also for ${ }^{3} \mathrm{He}$ photodisintegration the nuclear matrix element

$$
N_{3 \mathrm{~N}}^{\mu} \equiv\left\langle\Psi_{\text {scatt }}^{3 \mathrm{~N}}\left|j^{\mu}\right| \Psi_{\text {bound }}^{3 \mathrm{~N}}\right\rangle,
$$

is a crucial quantity. In our formalism, for the final state $\left|\Psi_{\text {scatt }}^{3 \mathrm{~N}}\right\rangle$ with three free nucleons, the $N_{3 \mathrm{~N}}^{\mu}$ is given by

$$
N_{3 \mathrm{~N}}^{\mu}=\left\langle\Phi_{3 \mathrm{~N}}\left|(1+P) j^{\mu}\right| \Psi_{\text {bound }}^{3 \mathrm{~N}}\right\rangle+\left\langle\Phi_{3 \mathrm{~N}}|(1+P)| U^{\mu}\right\rangle,
$$

where $P$ is the permutation operator, $P=P_{12} P_{23}+P_{13} P_{23}$, and $\left|\Phi_{3 \mathrm{~N}}\right\rangle$ is an antisymmetrized state describing the three outgoing nucleons. The auxiliary state $\left|U^{\mu}\right\rangle$ fulfills the Faddeev-like equation

$$
\left|U^{\mu}\right\rangle=t G_{0}(1+P) j^{\mu}\left|\Psi_{\text {bound }}^{3 \mathrm{~N}}\right\rangle+t G_{0} P\left|U^{\mu}\right\rangle,
$$

with $G_{0}$ being the free three-nucleon propagator.

We use the same electromagnetic current operator for the deuteron and ${ }^{3} \mathrm{He}$ photodisintegration processes. We build it from the single nucleon electromagnetic current, which is supplemented by 
the implicit many-body operators incorporated via the Siegert theorem [15]. In the following we denote this approach as "SNC+Siegert".

In the case of the muon capture reactions nuclear matrix elements have similar structure as shown above. The only differences can be found in the initial and final nuclear states and in the current operator. Muon capture on the deuteron leads to a final state with two neutrons (and a muon neutrino in its leptonic part), and for the ${ }^{3} \mathrm{He}$ we restrict ourselves to the reaction channel with ${ }^{3} \mathrm{H}$ and a muon neutrino in the final state. Using precalculated ${ }^{3} \mathrm{He}$ and ${ }^{3} \mathrm{H}$ bound states, there is no need to solve the Faddeev-like equation (2.4). Of course, instead of the electromagnetic current operator, the weak current has to be taken now into account. We take it as a single nucleon current supplemented by the dominant $(p / M)^{2}$ relativistic corrections, where $M$ is the nucleon mass. A detailed discussion of the weak current and the formulas connecting the nuclear matrix elements with the capture rates are given in [14], while our method to obtain $3 \mathrm{~N}$ bound states is presented in [19]. The technical steps required to compute the nuclear matrix elements, including the partial wave basis and decomposition of the operators present in Eqs. (2.1)-(2.5) can be found in [20].

\section{Results}

We use deuteron photodisintegration as a first test of application of improved chiral forces to electroweak processes. In Fig. 1 we show the total cross section for this process at photon laboratory energies $\mathrm{E}_{\gamma}$ up to $80 \mathrm{MeV}$. Our predictions employ the $\mathrm{N}^{4} \mathrm{LO}$ potential with cut-off parameter $\mathrm{R}=0.9 \mathrm{fm}$. The red line shows predictions based only on the SNC, while the dashed black curve corresponds to our full predictions (SNC+Siegert). The large effect of the (implicit and explicit) many-body currents is clearly seen and the experimental data are very nicely described by this model. For the sake of comparison with the predictions based on semi-phenomenological forces, we show also results obtained with the AV18 force [21] and different models of the nuclear current: the SNC (green curve), SNC+Siegert (dotted violet curve) and SNC+MEC (blue curve). In the latter model beside the SNC also dominant "seagull" and "meson-in-flight" currents are explicitly included, see $[15,17]$ for more details. The predictions based on the improved chiral force are in a good agreement with ones based on the AV18. In particular the SNC+Siegert predictions are practically indistinguishable at photon energies below approx. $30 \mathrm{MeV}$. At higher energies a small difference develops and the chiral predictions are even closer to the data of Ref. [26].

As examples of observables for ${ }^{3} \mathrm{He}$ photodisintegration we choose the semi-inclusive cross sections in three-body breakup, i.e. the process in which only one particle is detected. In each of Figs. 2-7 we show the cross section at photon laboratory energy $\mathrm{E}_{\gamma}=40 \mathrm{MeV}$ as a function of the final neutron or proton energy emerging at four angles $\Theta$ with respect to the photon beam: $\Theta=$ $0^{\circ}, 60^{\circ}, 120^{\circ}$ and $180^{\circ}$. The same structure of the spectra is observed for semi-phenomenological forces and is discussed in [27] and [17], so here we focus on the issues connected to using new forces. In Figs. 2 and 3 we show the convergence of predictions with respect to order of the chiral expansion for the detected neutron and proton, respectively. Only predictions at leading order (LO) are far away from the other ones and for sure are not sufficient to describe this process. The other predictions are close to each other. However at few angles, like $\Theta_{n}=0^{\circ}$ and $\mathrm{E}_{\mathrm{n}} \approx 18 \mathrm{MeV}$ or $\Theta_{p}=180^{\circ}$ and $\mathrm{E}_{\mathrm{p}} \approx 4 \mathrm{MeV}$, the action of chiral forces at higher orders are seen. Of course, we are aware that the final conclusions about the convergence pattern can be drawn only after consistent 


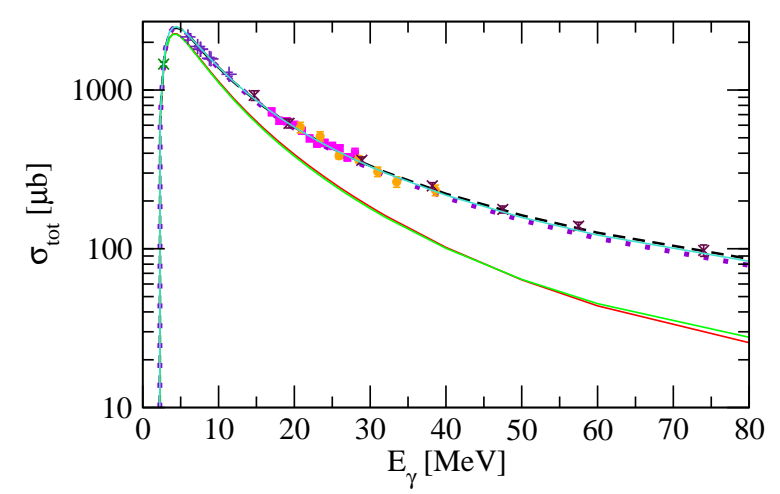

Figure 1: The total cross section for the $\gamma+\mathrm{d} \rightarrow \mathrm{p}+\mathrm{n}$ reaction. The chiral $\mathrm{N}^{4} \mathrm{LO}$ predictions for the SNC (SNC+Siegert) current model are shown with the red (black dashed) curve. The AV18 predictions for the SNC, SNC+Siegert and SNC+MEC current models are shown with the green, dotted violet and blue curve, respectively. The experimental data are from Ref. [22] (green x'es), [23] (magenta squares), [24] (orange dots), [25] (indigo pluses) and [26] (maroon x'es).

3NFs and current operators are included, but presented here predictions constitute a promising first step in this direction.
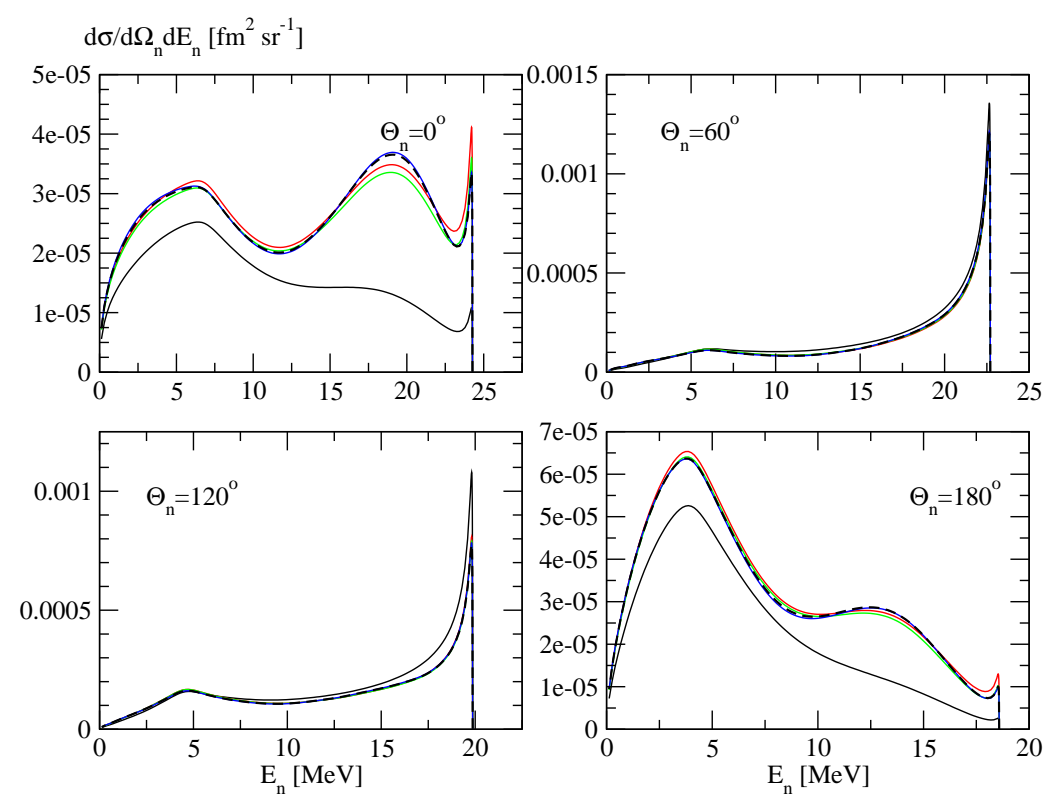

Figure 2: The semi-inclusive ${ }^{3} \mathrm{He}(\gamma, \mathrm{n}) \mathrm{pp}$ cross section at $\mathrm{E}_{\gamma}=40 \mathrm{MeV}$, as a function of the outgoing neutron energy for various laboratory angles. The predictions were obtained within the SNC+Siegert model and with the cut-off parameter $\mathrm{R}=0.9 \mathrm{fm}$. The solid black, red, green, blue and dashed black curves correspond to LO, NLO, $\mathrm{N}^{2} \mathrm{LO}, \mathrm{N}^{3} \mathrm{LO}$ and $\mathrm{N}^{4} \mathrm{LO}$ predictions, respectively.

To estimate the theoretical errors of predictions resulting from neglecting, at a given order of the chiral expansion, the contributions from the higher orders, we apply the prescription given in [9]. The resulting bands which correspond to the truncation errors are shown in Figs. 4 and 5. A big contribution from higher orders is expected at the next-to leading order (NLO) (the yellow band) and still noticeable, at the same nucleon energies, addition is expected at $\mathrm{N}^{2} \mathrm{LO}$ (the green 

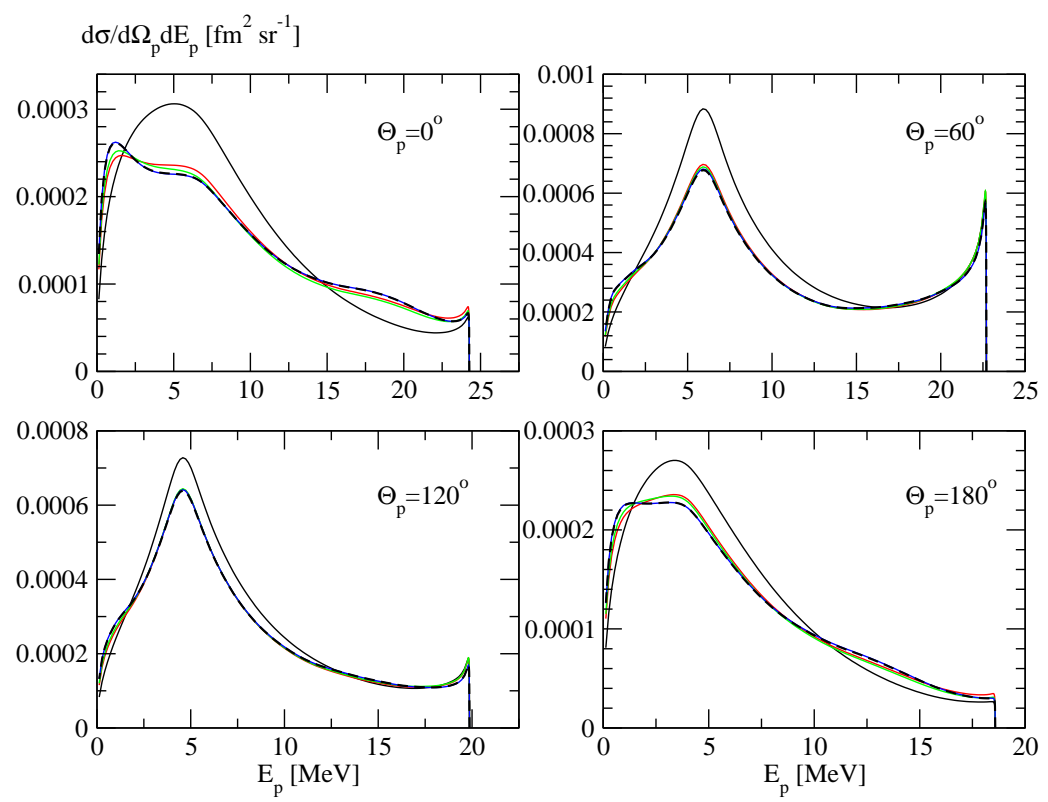

Figure 3: The same as in Fig. 2 but for the proton detection.

band). At higher orders bands are very narrow and practically overlap. Thus, one can conclude that for the presented here cross section calculations at $\mathrm{N}^{3} \mathrm{LO}$ should be sufficient.

Finally, in Figs.6 and 7 we show explicitly the dependence of the cross section on the value of the cut-off parameter $\mathrm{R}$ used to regularize the chiral forces at $\mathrm{N}^{4} \mathrm{LO}$. We use five different values $\mathrm{R}=0.8,0.9, \ldots, 1.2 \mathrm{fm}$ and observe very weak cut-off dependence. Only in some cases predictions at $\mathrm{R}=1.2 \mathrm{fm}$ lie slightly away from others. It can also be seen that the dispersion of predictions based on different values of $\mathrm{R}$ usually exceeds the theoretical uncertainties at $\mathrm{N}^{4} \mathrm{LO}$ shown in Figs. 4-5, but note that only values $\mathrm{R}=0.9 \mathrm{fm}$ and $\mathrm{R}=1.0 \mathrm{fm}$ are recommended $[7,8]$.

The results for two muon capture reactions, $\mu^{-}+\mathrm{d} \rightarrow \mathrm{n}+\mathrm{n}+v_{\mu}$ and $\mu^{-}+{ }^{3} \mathrm{He} \rightarrow{ }^{3} \mathrm{H}+v_{\mu}$, are given in Tabs. 1 and 2. It is clearly seen that the dependence on the cut-off parameter is very weak. The maximal difference between predictions at given order, $\Delta$, first increases when going from the LO to the NLO and then continously drops achieving at $\mathrm{N}^{4} \mathrm{LO} 0.44 \%$ for muon capture on the deuteron and $1.76 \%$ for the ${ }^{3} \mathrm{He}-{ }^{3} \mathrm{H}$ transition. For both processes we also observe the nice convergence of capture rates with respect to the chiral order. The new predictions are also in agreement with the results based on the AV18 NN force, which are $382.3 \mathrm{~s}^{-1}$ and 1295 $\mathrm{s}^{-1}$ for muon capture on the deuteron and for the non-breakup channel in muon capture on ${ }^{3} \mathrm{He}$, respectively.

\section{Summary}

Recently developed chiral forces with local regularization were applied to study the deuteron and ${ }^{3} \mathrm{He}$ photodisintegration reactions as well as the muon capture processes on the deuteron and ${ }^{3} \mathrm{He}$. The Siegert theorem was used to include many-body currents for the photodisintegration reactions and on top of the single nucleon current the relativistic corrections were taken into account for the weak processes. For all investigated reactions we could confirm the good behavior of the 

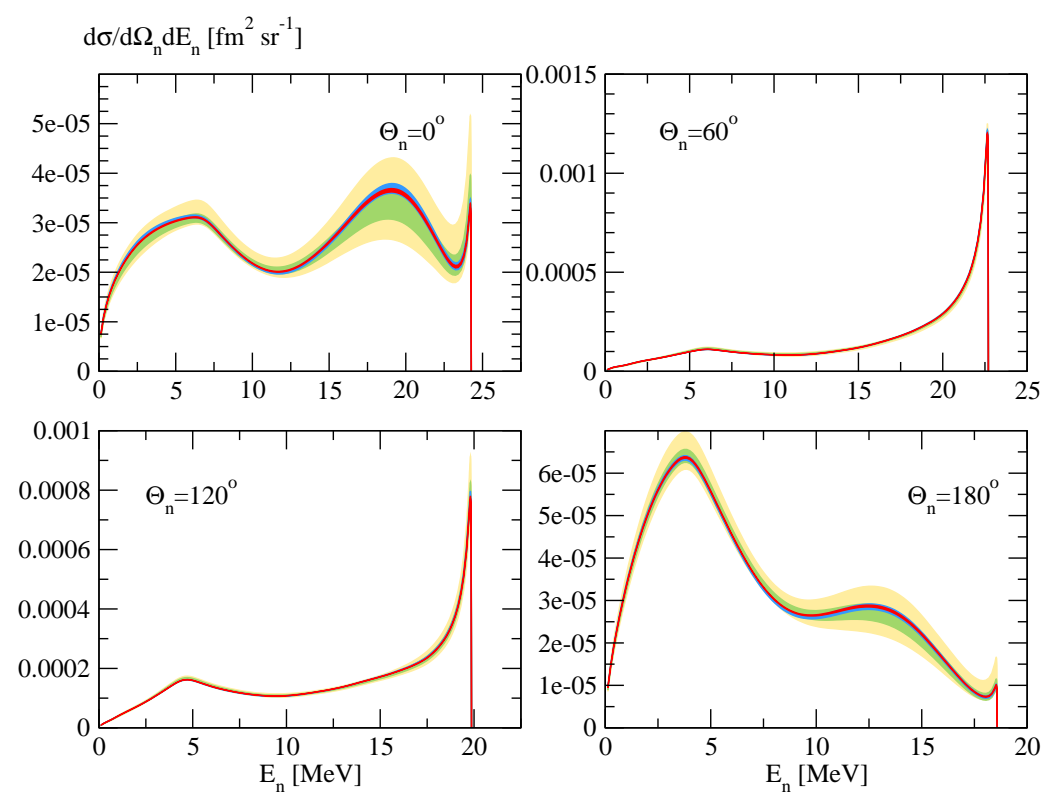

Figure 4: The estimated higher order truncation errors for the cross sections shown in Fig. 2. The yellow, green, blue and red bands show the theoretical uncertainties at NLO, $\mathrm{N}^{2} \mathrm{LO}, \mathrm{N}^{3} \mathrm{LO}$ and $\mathrm{N}^{4} \mathrm{LO}$, respectively.
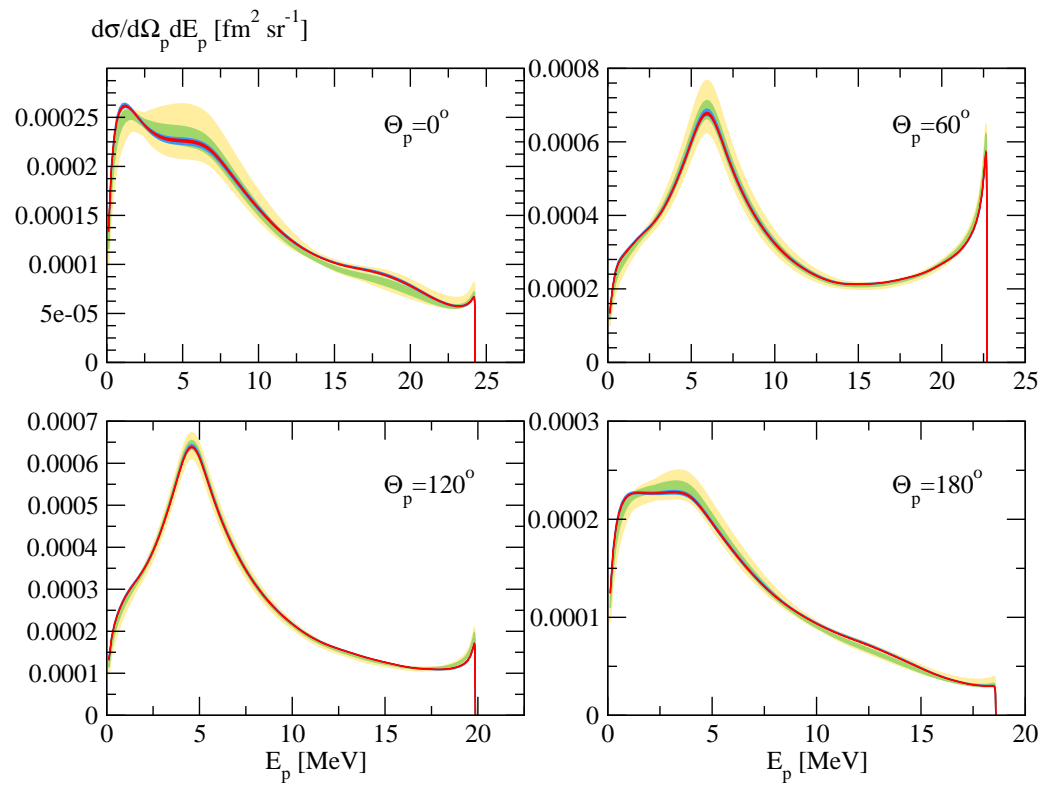

Figure 5: The same as in Fig. 4 but for the proton detection. 

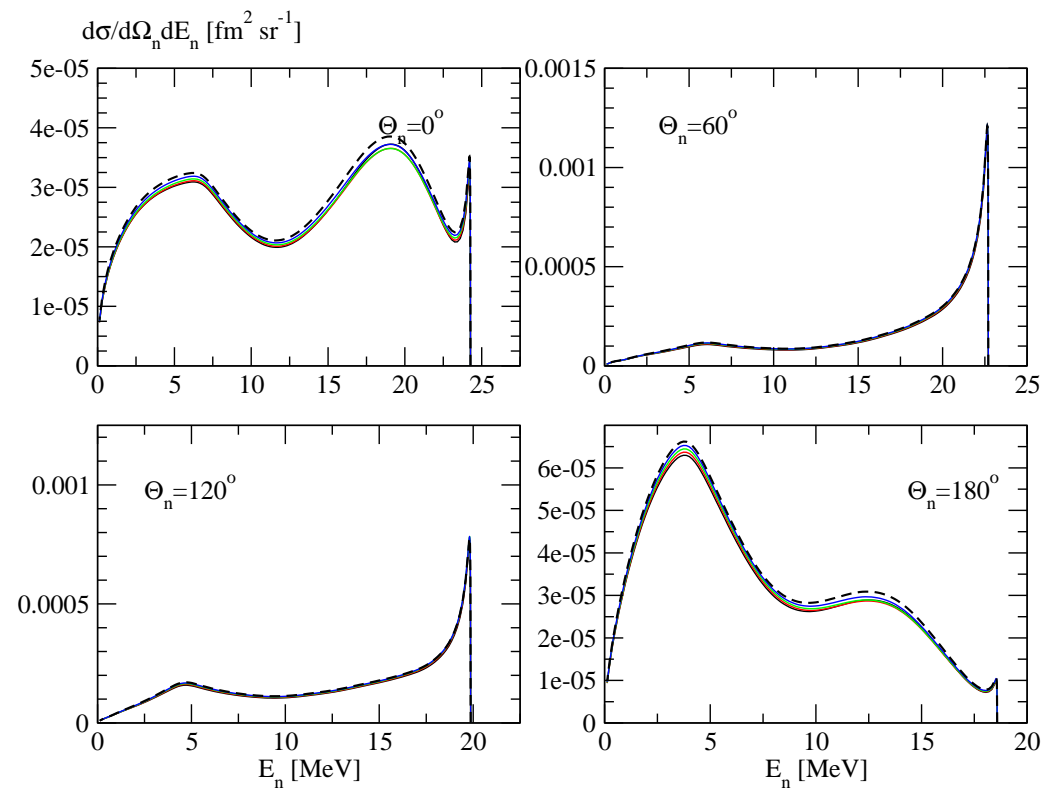

Figure 6: The cut-off dependence of the cross sections shown in Fig. 2. The solid black, red, green and blue curves are for $\mathrm{R}=0.8,0.9,1.0,1.1 \mathrm{fm}$, respectively, and the dashed black curve represents predictions for $\mathrm{R}=1.2 \mathrm{fm}$.
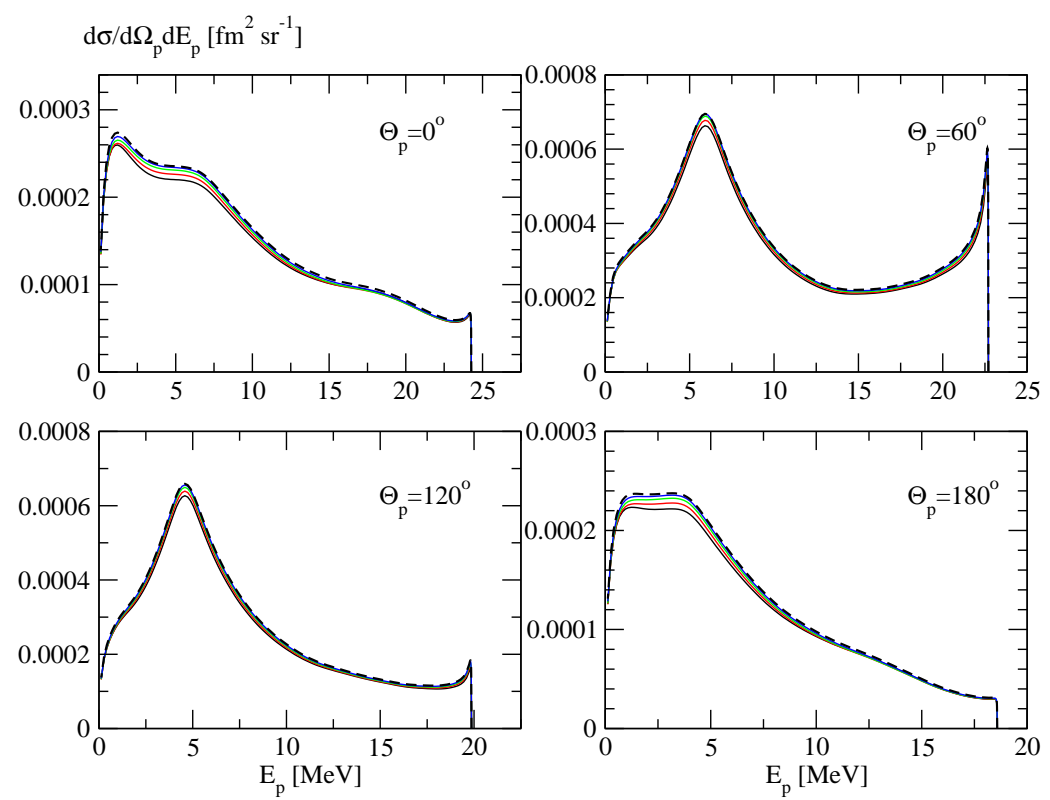

Figure 7: The same as in Fig. 6 but for the proton detection. 
Study of electroweak processes in the two and three-nucleon systems with local chiral forces Roman Skibinski

\begin{tabular}{|c|c|c|c|c|c|c|}
\hline chiral order & $\mathrm{R}=0.8 \mathrm{fm}$ & $\mathrm{R}=0.9 \mathrm{fm}$ & $\mathrm{R}=1.0 \mathrm{fm}$ & $\mathrm{R}=1.1 \mathrm{fm}$ & $\mathrm{R}=1.2 \mathrm{fm}$ & $\Delta$ \\
\hline LO & 396.0 & 397.4 & 398.4 & 398.9 & 399.2 & 3.3 \\
NLO & 384.2 & 385.8 & 387.2 & 388.6 & 389.8 & 5.7 \\
N2LO & 385.0 & 386.1 & 387.2 & 388.3 & 389.3 & 4.3 \\
N3LO & 386.8 & 386.4 & 385.2 & 384.3 & 383.2 & 3.6 \\
N4LO & 385.5 & 386.1 & 386.3 & 385.6 & 384.6 & 1.7 \\
\hline
\end{tabular}

Table 1: The doublet capture rates in $\left[\mathrm{s}^{-1}\right]$ for the $\mu^{-}+\mathrm{d} \rightarrow \mathrm{n}+\mathrm{n}+v_{\mu}$ process obtained within the $\mathrm{SNC}+\mathrm{RC}$ model of weak current (see text). In the last column the spread of the results at a given chiral order due to different $\mathrm{R}$ values, $\Delta$ in $\left[\mathrm{s}^{-1}\right]$, is shown.

\begin{tabular}{|c|c|c|c|c|c|c|}
\hline chiral order & $\mathrm{R}=0.8 \mathrm{fm}$ & $\mathrm{R}=0.9 \mathrm{fm}$ & $\mathrm{R}=1.0 \mathrm{fm}$ & $\mathrm{R}=1.1 \mathrm{fm}$ & $\mathrm{R}=1.2 \mathrm{fm}$ & $\Delta$ \\
\hline $\mathrm{LO}$ & 1610 & 1618 & 1610 & 1594 & 1572 & 46 \\
$\mathrm{NLO}$ & 1330 & 1357 & 1381 & 1405 & 1427 & 97 \\
$\mathrm{~N} 2 \mathrm{LO}$ & 1337 & 1356 & 1376 & 1395 & 1415 & 78 \\
$\mathrm{~N} 3 \mathrm{LO}$ & 1314 & 1304 & 1289 & 1278 & 1266 & 48 \\
N4LO & 1296 & 1307 & 1308 & 1299 & 1285 & 23 \\
\hline
\end{tabular}

Table 2: The total capture rates in $\left[\mathrm{s}^{-1}\right]$ for the $\mu^{-}+{ }^{3} \mathrm{He} \rightarrow{ }^{3} \mathrm{H}+v_{\mu}$ process obtained within the $\mathrm{SNC}+\mathrm{RC}$ model of weak current (see text). In the last column the spread of the results at a given chiral order due to different $\mathrm{R}$ values, $\Delta$ in $\left[\mathrm{s}^{-1}\right]$, is shown.

used NN interaction. Namely, we observed fast convergence of the predictions with respect to the order of the chiral expansion - for all studied observables predictions at $\mathrm{N}^{4} \mathrm{LO}$ are very close to ones at $\mathrm{N}^{3} \mathrm{LO}$. We observe also very weak dependence of our results on the value of the local regulator parameter R. Especially, predictions with the most recommended values $R=0.9 \mathrm{fm}$ and $R=1.0 \mathrm{fm}$ usually overlap at $\mathrm{N}^{4} \mathrm{LO}$. The observed cut-off dependence is much weaker than the one present for the older chiral forces, which were regularized directly in the momentum space, using the nonlocal regulators. Finally, we show estimates for the truncation errors coming from neglecting of the higher order contributions. These theoretical uncertainties decrease with the higher and higher chiral order and are very small above $\mathrm{N}^{2} \mathrm{LO}$. This shows the importance of the improved chiral potentials for few-body investigations. However, it is necessary to verify these conclusions for other observables and at different, especially higher, energies. Such work is in progress.

\section{References}

[1] R.Machleidt and D.R. Entem, Chiral effective field theory and nuclear forces, Phys.Rept. 503 (2011) 1.

[2] E.Epelbaum et al., Modern theory of nuclear forces, Rev.Mod.Phys. 81 (2009) 1773.

[3] Jiunn-Wei Chen et al., Nucleon-nucleon effective field theory without pions, Nucl.Phys. A653 (1999) 386.

[4] H.Krebs et al., Nuclear forces with $\Delta$ excitations up to next-to-next-to-leading order, part I: Peripheral nucleon-nucleon waves, Eur.Phys.J. A32 (2007) 127.

[5] E.Epelbaum et al., The two-nucleon system at next-to-next-to-next-to-leading order, Nucl.Phys. A747 (2005) 362. 
[6] H.Witała et al., Calculations of three-nucleon reactions with $N^{3} L O$ chiral forces: achievements and challenges, J.Phys. G41 (2014) 094011.

[7] E.Epelbaum et al., Improved chiral nucleon-nucleon potential up to next-to-next-to-next-to-leading order, Eur.Phys.J. A51 (2015) 53.

[8] E.Epelbaum et al., Precision Nucleon-Nucleon Potential at Fifth Order in the Chiral Expansion, Phys.Rev.Lett. 115 (2015) 122301.

[9] S.Binder et al, Few-nucleon systems with state-of-the-art chiral nucleon-nucleon forces, arXiv: 1505.07218 .

[10] S.Kölling et al., Two-nucleon electromagnetic current in chiral effective field theory: One-pion exchange and short-range contributions, Phys.Rev. C84 (2011) 054008.

[11] D.Rozpędzik et al., Signatures of the chiral two-pion exchange electromagnetic currents in the ${ }^{2} H$ and ${ }^{3}$ He photodisintegration reactions, Phys.Rev. C83 (2011) 064004.

[12] R.Skibiński et al., The Chiral Long-range Two-pion Exchange Electromagnetic Currents in Radiative Nucleon-Deuteron Capture, Acta Phys.Polon. B46 (2015) 159.

[13] R.Skibinski et al., Nucleon-deuteron capture with chiral potentials, Acta Phys.Polon. B37 (2006) 2905.

[14] J.Golak et al., Break-up channels in muon capture on ${ }^{3} H e$, Phys. Rev. C90 (2014) 024001.

[15] J.Golak et al., Faddeev calculations of proton deuteron radiative capture with exchange currents, Phys.Rev. C62 (2000) 054005.

[16] R.Skibiński et al., Search for three nucleon force effects in two-body photodisintegration of ${ }^{3} \mathrm{He}\left({ }^{3} \mathrm{H}\right)$ and in the time reversed proton deuteron radiative capture process, Phys.Rev. C67 (2003) 054001.

[17] J.Golak et al., Electron and photon scattering on three-nucleon bound states, Phys.Rept. 415 (2005) 89.

[18] R.Skibinski et al., Different formulations of ${ }^{3}$ He and ${ }^{3}$ H photodisintegration, Eur.Phys.J. A24 (2005) 31.

[19] A.Nogga et al., Benchmark calculations for the triton binding energy for modern NN forces and the $\pi \pi$ exchange three nucleon force, Phys.Lett. B409 (1997) 19.

[20] W.Glöckle et al., The three-nucleon continuum: achievements, challenges and applications, Phys.Rept. 274 (1996) 107.

[21] R.B.Wiringa et al., An Accurate nucleon-nucleon potential with charge independence breaking, Phys.Rev. C51 (1995) 38.

[22] R.Moreh et al., ${ }^{2} H(\gamma, n)$ absolute cross section at 2754 keV, Phys.Rev. C39 (1989) 1247.

[23] D.M.Skopik et al., Photodisintegration of deuterium determined from the electrodisintegration process, Phys.Rev. C9 (1974) 531.

[24] M.Bosman et al., Measurement of the total cross section for the ${ }^{1} H(n, \gamma){ }^{2} H$ reaction between 37 and 72 MeV, Phys.Lett. B82 (1979) 212.

[25] Y.Birenbaum et al., Absolute cross section for the photodisintegration of deuterium, Phys.Rev. $\mathbf{C 3 2}$ (1985) 1825.

[26] R.Bernabei et al., Deuteron Photodisintegration Total Cross-section Between $15 \mathrm{MeV}$ and $75 \mathrm{MeV}$, Phys.Rev.Lett. 57 (1986) 1542.

[27] R.Skibiński et al., Three-nucleon photodisintegration of ${ }^{3}$ He, Phys.Rev. C67 (2003) 054002. 